\title{
Papel de los inhibidores de la absorción de la glucosa SGLT2 en el tratamiento de la Diabetes Mellitus tipo 2
}

\author{
Role of the sodium-glucose linked transporter 2 (SGLT2) \\ inhibitors in the treatment of Diabetes Mellitus type 2
}

Dante Américo Gamarra-González ${ }^{1}$

Resumen

Se revisa la fisiopatología renal de la diabetes mellitus tipo 2 (DM2). Se analiza el enfoque que rompe el paradigma de tratar al paciente diabético solo mediante el control de la glicemia. Se revisa el mecanismo de acción de los llamados cotransportadores de sodio-glucosa2 (SGLT2), sus indicaciones, seguridad cardiovascular, efectos pleiotrópicos, los potenciales efectos adversos y su papel en disminuir la severidad y mortalidad por complicaciones cardiovasculares y renales. Los tres principales inhibidores de SGLT2 (dapagliflozina, canagliflozina y empagliflozina) han demostrado eficacia como monoterapia o en combinación con insulina o con otros hipoglicemiantes orales en el tratamiento de la DM2. Provocan pérdida significativa de peso, efecto positivo sobre la presión arterial y producen cambios del perfil lipídico.

Palabras clave: Diabetes Mellitus tipo 2, tratamiento, inhibidores de la SGLT2.

Abstract

A review of renal physiopathology of diabetes mellitus type 2 (DM2) is performed. The paradigmatic focusing of the DM2 treatment by means only on the glycemic control is analyzed. A review of mechanism of action of the so called sodium-dependent glucose cotransporters inhibitors type 2 (SGLT2) is done as well as its indications, cardiovascular safety, pleiotrophic effects, potential side effects and their role in dismissing the severity and mortality due cardiovascular and renal complications. The three main STLT2 inhibitors (dapagliflozin, canagliflozin and empagliflozin) have demonstrated efficacy in the treatment of DM2 both as mono therapy as well as in combination with insulin and/or oral hypoglycemic agents. They produce a significant weight loss, beneficial effects on blood pressure and changes in lipid profile.

Keywords: Diabetes Mellitus type 2, treatment, SGLT2 inhibitors.

Introducción

La Diabetes Mellitus tipo 2 es una de las enfermedades más prevalentes a nivel mundial, con cerca de 390 millones de personas afectadas y que lamentablemente el $45 \%$ de ellas; no son diagnosticados tempranamente. Este porcentaje de pacientes se diagnostican años después presentando manifestaciones crónicas $^{(1)}$.

El desafió actual es la intervención más temprana posible del control de la glicemia para evitar las complicaciones microvascular y macrovasculares.
Los estimados nacionales e internacionales muestran una prevalencia de más del $60 \%$ de pacientes atendidos en hospitales con edad mayor a los 65 años y comorbilidades asociadas a la diabetes como hipertensión arterial, obesidad, ateroesclerosis, insuficiencia cardíaca y enfermedad renal crónica $^{(2)}$.

En consecuencia, en los pacientes de edad avanzada la atención no solo reside en controlar los niveles de la glucosa sino también las comorbilidades acompañantes, teniendo en cuenta los efectos adversos de medicamentos y la hipoglucemia, que incrementan el riesgo de un desenlace fatal.

${ }^{1}$ Jefe del Servicio de Endocrinología, Hospital Nacional Dos de Mayo, MINSA, Lima - Perú. Maestría en Docencia e Investigación en Salud. Facultad de Medicina Universidad Nacional Mayor de San Marcos (UNMSM). Docente Tutor de Residentado de Endocrinología Facultad de Medicina UNMSM, Docente Pre Grado, Facultad de Medicina, Universidad Particular San Martín de Porres y Facultad de Medicina, Universidad Ricardo Palma. Delegado electo de Perú ante la Asociación Latinoamericana de Diabetes (ALAD). ORCID N ${ }^{\circ} 0000-0002-1166-7121$. 


\section{Cuerpo de Trabajo}

\section{Homeostasis renal de la glucosa}

En condiciones normales, el riñón cumple un papel importante en la homeostasis de la glucosa mediante tres mecanismos reguladores: gluconeogénesis, utilización de la glucosa y reabsorción de la glucosa filtrada. El $90 \%$ de la glucosa se reabsorbe en el túbulo proximal mediante los llamados co-transportadores de sodio-glucosa2 (SGLT2). Se ha calculado que hasta $180 \mathrm{gr}$ de glucosa se reabsorben al día, por lo que en condiciones normales no se excreta glucosa en la orina $^{(3,4)}$.

En los pacientes diabéticos mal controlados, se ha observado alteración del ARNm de los SGLT2, que conduce a un incremento del número y actividad de los receptores de los co-transportadores; elevando el umbral máximo de la reabsorción de glucosa $\mathrm{y}$, finalmente un incremento de la hiperglucemia ${ }^{(3)}$.

En ese contexto, las primeras investigaciones sobre medicamentos que pudieran actuar sobre este mecanismo fisiopatológico de la diabetes mellitus tipo 2 , se realizaron con la florizina. Una sustancia que se encuentra en la raíz de la corteza de los árboles fruteros y que posee un efecto inhibidor competitivo natural de los SGLT1 y SGLT2, con mayor efectividad por el segundo ${ }^{(3)}$.

Las primeras investigaciones se realizaron en la década de los 80 con la florizina, en animales de experimentación (en ratas se producía una normalización de la glucosa sin producir hipoglucemia). La metabolización de la florizina en floritina, la hace poco biodisponible para su empleo clínico ${ }^{(3)}$.

Los derivados de la florizina que contienen un componente c-glucósido le proporciona resistencia a la degradación enzimática. Los más estudiados y utilizados con eficacia y seguridad comprobada son dapagliflozina, canagliflozina y empagliflozina, que han sido aceptadas por la Administración de Alimentos y Medicamentos (FDA) y la Agencia Europea de Medicamentos (EMA) $)^{(4)}$.

Otros medicamentos de la misma clase más recientes de SGLT2 como ipragliflozina, luseogliflozina y tofogliflozina, están disponibles para su uso en Japón, donde el proceso de aprobación de medicamentos es más flexible en seguridad a largo plazo.

También existen otros inhibidores de la SGLT2 en varias etapas de desarrollo, como la ertugliflozina que ha entrado en el desarrollo de la Fase III para el tratamiento de la diabetes tipo $2^{(5)}$ y la sotagliflozina, un inhibidor combinado de SGLT1 y SGLT2, se ha estudiado en la diabetes tipo $1^{(6)}$.

\section{Mecanismo de acción}

El mecanismo de acción es inhibir la acción de los SGLT2 en los riñones, reduciendo la reabsorción de glucosa en el túbulo contorneado proximal y aumentando la excreción de glucosa en la orina. La glucosa excretada en la orina equivale a una pérdida neta de 200 a $300 \mathrm{kcal} /$ día y la reducción de peso es un efecto secundario favorable (Figura 1).

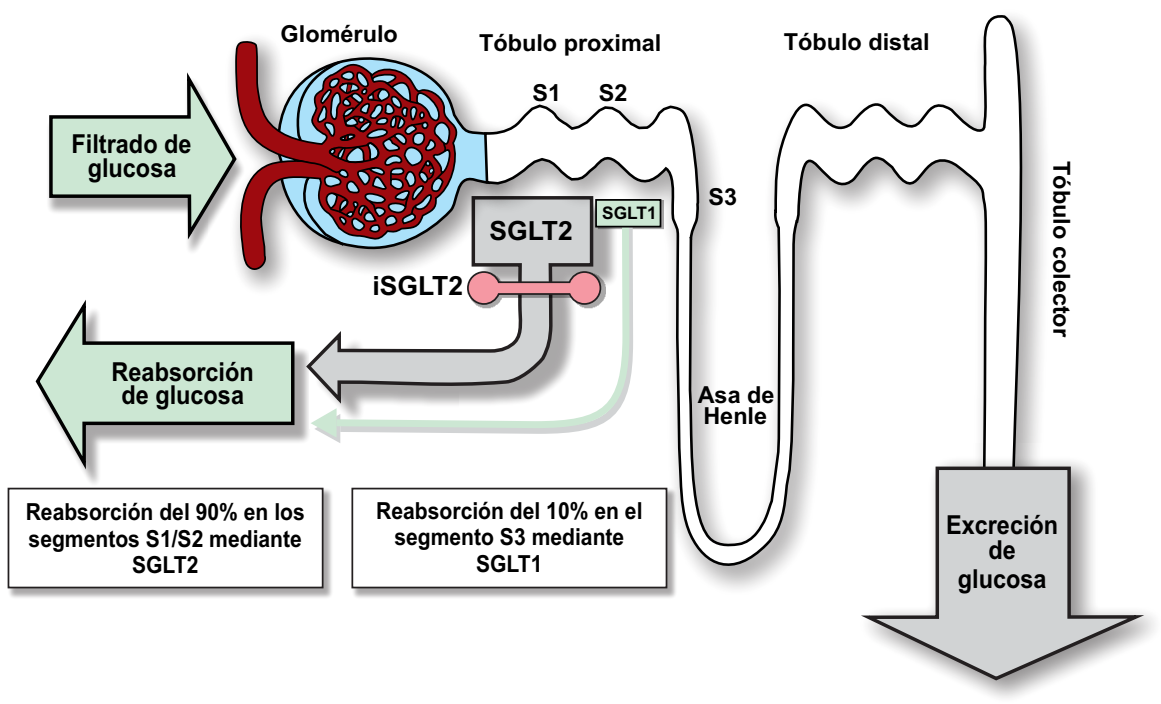

Figura 1. Mecanismo de acción de los inhibidores SGT2 en el control de la glucosa Tomado de: Rev Esp Cardiol.2016:1088-1097. 


\section{Efectos en el control de la glucosa}

El efecto del control de la glucosa con la dapagliflozina, se evidenció en 12 estudios clínicos que incluyo 6,998 pacientes diabéticos tipo 2; con $\mathrm{HbAlc}$ en un rango de 7 a $10 \%$, comparado con dos grupos control: uno con placebo y el otro con metformina. Se mejoró significativamente el control glucémico con cambios de la HbAlc de -0.23 a $-0.89 \%$, comparada con el grupo placebo con las dosis de $2.5,5$ y $10 \mathrm{mg}$ de dapagliflozina, respectivamente ${ }^{(7,8)}$. En el grupo paralelo con metformina la dapagliflozina mejoró significativamente el control glucémico con cambios medios de HbAlc de $(-0.3 \%$ con placebo, $-0.67 \%$ con $5 \mathrm{mg},-0,7 \%$ con $10 \mathrm{mg}^{(9,10)}$.

El control de la glucosa se ha reportado en otros estudios, cuyos hallazgos son similares cuando se adiciona glipizida, glimepirida (sulfonilureas) y sitagliptina (inhibidor de la DPP-4) $)^{(11,12)}$.

Los efectos de control de glucosa con la canagliflozina, se evidenciaron en estudios que incluyeron a 7,725 pacientes diabéticos comparados con placebo; la canagliflozina a dosis de $100 \mathrm{mg}$ a $300 \mathrm{mg}$ redujo significativamente la $\mathrm{HbAlc}$ en comparación con el placebo $(-0.77,-1.03$ y $0.14 \%$, respectivamente $)^{(13,14)}$.

Para la empagliflozina, se incluyó siete estudios con 5306 pacientes con diabetes. En ensayos aleatorizados en los que compararon con sitagliptina y metformina; se encontró una disminución de la hemoglobina glicosilada entre -0.13 a $-0.7 \%$, con dosis de empagliflozina $10 \mathrm{mg} \mathrm{y} 20 \mathrm{mg}^{(15,16)}$ (Tabla 1 ).

\section{Efectos sobre el peso}

Los estudios con empagliflozina y dapagliflozina en relación al peso mostraron cambios significativos. En un estudio de 24 semanas utilizando dapagliflozina como terapia inicial versus el grupo control (bajas dosis de insulina) tuvieron una pérdida de peso de entre $-2.2 \mathrm{~kg}$ y $-3.3 \mathrm{~kg}$, respectivamente; a predominio de grasa corporal magra ${ }^{(17,18)}$.

Adicionalmente a la disminución de peso, se ha demostrado que la dapagliflozina reduce significativamente la circunferencia abdominal $(-1.52 \mathrm{~cm})$ en comparación con el grupo control ${ }^{(18)}$. En este estudio, aproximadamente dos tercios de la pérdida de peso se atribuyeron a reducciones en la masa $\operatorname{grasa}^{(18)}$.

En el estudio de dapagliflozina versus placebo, el grupo control recibió insulina, cuyo cambio de peso fue $+0.43 \mathrm{~kg}, \mathrm{y}$ al agregar insulina al grupo de dapagliflozina el peso disminuyó en $-2.04 \mathrm{~kg}^{(19)}$.

En otro estudio utilizando metformina versus placebo, al añadir dapagliflozina en todas sus presentaciones al grupo de metformina, mostraron una pérdida sostenible de peso (de $1.10 \mathrm{~kg} \mathrm{a}-1.74 \mathrm{~kg}$ ), en comparación con su aumento en el grupo control $^{(20)}$

\section{Presión arterial}

Debido a la diuresis osmótica crónica por la glucosuria (aumento de orina en 24 horas de $107 \mathrm{a} 400 \mathrm{ml}$.) provocada por

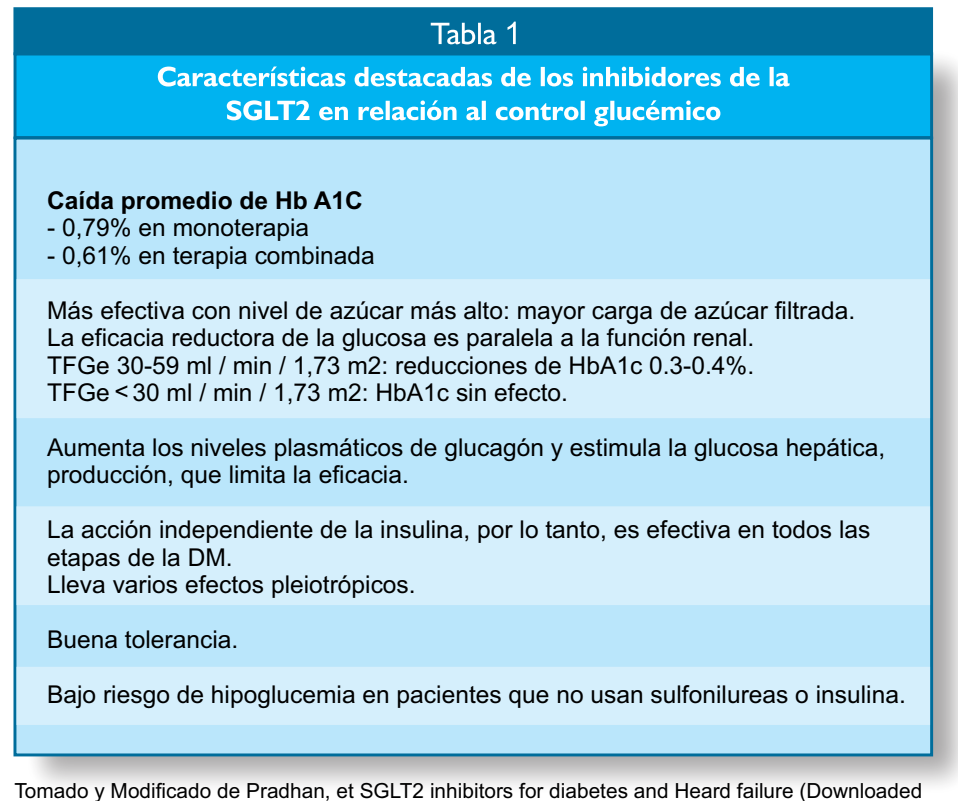

free from htpp //www.jfmpc.com on Saturday, February 22.2020. IP: 189.163.121.31 
los inhibidores de la SGLT2. Se observa una reducción de la presión arterial sistólica. En 12 estudios con dosis de $10 \mathrm{mg}$ de dapagliflozina, la presión arterial sistólica se redujo entre 2 y 9 $\mathrm{mm}$ de $\mathrm{Hg}$ sin tener un aumento de la frecuencia cardíaca ni episodios de síncope ${ }^{(21)}$.

Con la empagliflozina se observó resultados similares, mostrando una reducción en la presión arterial sistólica que fue significativa en comparación con el placebo. Se observó una mayor reducción en hipertensos, siendo de 2.6 y $3.3 \mathrm{~mm} \mathrm{Hg}$ con las dosis de $10 \mathrm{mg}$ y $25 \mathrm{mg}^{(22)}$; mostrando que los efectos antihipertensivos son independientes de su capacidad de hacer perder peso y mejorar el control de glucosa ${ }^{(22)}$.

\section{Efectos Adversos y Seguridad}

\section{Hipoglucemia}

Los inhibidores de SGLT2 son bien tolerados y se han usado de manera segura cuando se prescriben como monoterapia o en combinación con otros agentes hipoglucemiantes orales o terapia con insulina.

No se han documentado episodios importantes de hipoglucemia con el uso de los inhibidores de SGLT2 como monoterapia (estudios de Fase III). Además un estudio de metaanálisis concluyó que el riesgo de hipoglucemia era similar al asociado con otros agentes hipoglicemiantes como la metformina $^{(23)}$.

El riesgo de hipoglucemia aumenta cuando estos agentes se usan en combinación con sulfonilurea o terapia con insulina, en el contexto de enfermedad renal crónica (ERC) y en el tratamiento de pacientes de edad avanzada ${ }^{(24)}$.

Se aconseja usar dosis más bajas de insulina o de secretagogos de insulina (sulfonilureas) cuando se prescriben estas asociaciones con un inhibidor de SGLT2 ${ }^{(25)}$.

Se demostró evidencias que apoyan el uso de inhibidores de DPP4 en lugar de las sulfonilureas para la reducción de la episodios de hipoglucemia sin desmedro del control de peso y la diabetes $^{(26)}$.

\section{Infecciones Genitales}

Los pacientes diabéticos en general tienen un mayor riesgo de infección genital y del tracto urinario (ITU), debido a la hiperglucemia y la posterior glucosuria. El efecto de los inhibidores de SGLT2 induce glucosuria, por lo que se ha reportado un aumento asociado de infecciones genitales y de las vías urinarias. Varios ensayos controlados comparados con placebo informaron que las infecciones genitales y del tracto urinario fueron más comunes con la dapagliflozina, (infección genital 4.1-5.7\% vs. 0.9\%; e infección urinaria 3.6-5.7\% vs. $3.7 \%)^{(27)}$. Hallazgos similares se ha encontrado con el uso de canagliflozina (infección genital $7.5 \%$ vs. $1.9 \%$ en placebo e infección urinaria $5.1 \%$ vs. $4.0 \%$ placebo $)^{(27)}$.

Los datos para empagliflozina son similares para las infecciones del tracto genital, pero las diferencias no fueron estadísticamente significativas en cuanto a las infecciones urinarias ${ }^{(27)}$. En relación al género dichas infecciones fueron más frecuentes en mujeres.

\section{Hipovolemia}

Se produce diariamente cerca de $375 \mathrm{ml}$ de orina adicional en los pacientes que usan los inhibidores de la SGLT2, que se asocia a hipovolemia en mayores de 75 años, y en la utilización concomitante de diuréticos de asa en la enfermedad renal avanzada.

Otros estudios han demostrado aumentos clínicamente significativos en el hematocrito, urea sérica y creatinina, sin aumento en las tasas de insuficiencia renal, hipotensión o deshidratación ${ }^{(28)}$.

Los eventos de hipovolemia ocurrieron en 1.2 y $1.3 \%$ de los pacientes tratados con canagliflozina a dosis de $100 \mathrm{mg}$ y $300 \mathrm{mg}$ respectivamente, y no hubo eventos significativos con empagliflozina ${ }^{(29)}$.

\section{Efectos en pacientes con insuficiencia cardíaca}

El primer estudio EMPA-REG con empagliflozina reporta, en pacientes con diabetes tipo 2 y alto riesgo de evento cardiovascular, una menor tasa de mortalidad general y de hospitalización por insuficiencia cardíaca ${ }^{(30)}$, sugiriendo que los inhibidores de la SGLT2 tienen efectos pleiotrópicos sobre los parámetros hemodinámicos y metabólicos ${ }^{(30)}$.

El estudio de evaluación cardiovascular de Canagliflozina (CANVAS) evaluó la eficacia, seguridad y durabilidad en más de 10,000 pacientes con diabetes tipo 2, que tenían antecedentes de enfermedad cardiovascular o al menos dos factores de riesgo cardiovasculares. Los resultados mostraron que hubo una reducción de eventos $\mathrm{CV}$ y el infarto de miocardio no fatal (26.9 vs. $31.5 \%$ ); sin embargo, se descubrió un aumento del riesgo de amputación de miembros inferiores, un resultado corroborado en los estudios CANVAS y CANVAS- $\mathrm{R}^{(31)}$. 
En el ensayo DECLARE TIMI $58^{(32)}$, se utilizó a la Dapagliflozina, para evaluar el perfil de seguridad y efecto cardiovascular. Se evaluaron 17,160 pacientes, incluidos 10,186 sin enfermedad CV aterosclerótica, en un seguimiento de 4,2 años. El ensayo concluyó que, en pacientes con diabetes tipo 2 que tenían o estaban en riesgo de enfermedad $\mathrm{CV}$ aterosclerótica, el tratamiento con dapagliflozina no resultó en una tasa más alta o más baja de presencia de eventos cardiovasculares que el placebo, pero sí resultó en una tasa más baja de muerte por enfermedad cardiovascular o insuficiencia cardíaca $^{(32,33)}$.

El estudio CVD-REAL sobre la efectividad de los resultados cardiovasculares en nuevos usuarios de los inhibidores SGLT2 comparados con usuarios de otros tratamientos; comprendió a 309,056 pacientes, teniendo 154,528 pacientes en cada grupo ${ }^{(34)}$. En el tiempo total del estudio, la administración del fármaco correspondió al 53\% para canagliflozina, $42 \%$ para dapagliflozina y $5 \%$ para empagliflozina. Se asoció con tasas más bajas de insuficiencia cardíaca fatal y muerte sin significancia étnicas en los países donde se realizaron los estudios. Se concluye que el tratamiento con los inhibidores SGLT-2 versus otros medicamentos utilizados para reducir la glucosa se asoció con menor riesgo de falla cardíaca y muerte ${ }^{(35)}$.

\section{Efectos sobre la Función Renal}

Un estudio sobre dapagliflozina y placebo en pacientes con insuficiencia renal moderada con una tasa de filtración glomerular estimada entre 30 a $59 \mathrm{ml} / \mathrm{min} / 1.73 \mathrm{~m} 2$, no mostraron diferencias significativas en los resultados de $\mathrm{HbAlc}$, lo que confirma que para la eficacia de los inhibidores de SGLT2; se requiere una carga adecuada de filtración de glucosa a través de los túbulos renales. Las concentraciones plasmáticas de dapagliflozina y sus metabolitos aumentaron gradualmente al disminuir la función renal ${ }^{(36)}$.

En otro estudio se ha demostrado que las concentraciones plasmáticas de dapagliflozina fueron más altas en individuos con insuficiencia renal leve (4\%), moderada (6\%) y grave $(9 \%)$, en comparación con pacientes con la función renal normal $^{(37)}$.

\section{Cetoacidosis}

Diversos estudios han encontrado que un grupo de pacientes usuarios de inhibidores de SGLT2 podrían presentar riesgo de cetoacidosis diabética, incluso con valores de glucosa en ayunas y posterior a alimentos normales. Ello debido a la persistente glucosuria y la reducción de la producción de insulina por las células beta y un incremento del glucagón.

En un estudio comparativo de pacientes que utilizaron inhibidores de SGLT2, se presentaron cetoacidosis en 12 de 17,596 y 2 de 6,909 en los paciente que no la utilizaron.

Este estudio demostró un riesgo mayor de 7 veces de presentar citoacidosis en pacientes que utilizan inhibidores de la SGLT2. Por lo que se recomienda analizar mejor la situación clínica de los pacientes antes de utilizarlos que tengan glicemia normal con cuerpos cetónicos asociada a acidosis metabólica en ausencia de elevaciones de uremia o intoxicación exógena por otras sustancias ${ }^{(38)}$

La tabla 2 muestra un resumen de todos los efectos benéficos de los inhibidores de la la SGLT2.

Posicionamiento Actual en las

Guías de Tratamiento de la DM2

Después de la revisión sobre los mecanismos de acción $y$ los diversos efectos no solo en el control de la glucosa; sino en el retardo de la progresión de las manifestaciones cardiovasculares y renales, las diversas guías de tratamiento las muestran como una gran alternativa para el adecuado control de la diabetes mellitus tipo 2 .

\section{Conclusiones}

Los inhibidores de los SLGT2 son los medicamentos orales más recientes en el tratamiento actual de la diabetes mellitus tipo 2, estando considerados en los principales consensos dwe las asociaciones internacionales (ADA, AACE, IDF, ALAD). Por su impacto en el control de la diabetes y la disminución en la progresión de las complicaciones cardiovasculares y renales; brindando una mayor seguridad en el tratamiento con menor riesgo de hipoglucemia.

Es importante que se incorpore este grupo de fármacos a las guías de tratamiento en todas las instituciones del sistema de salud de nuestro país, y que sea utilizado en pacientes que presentan complicaciones cardíacas y renales, lo que impactaría en la disminución de la morbimortalidad que es altamente prevalente en los pacientes con diabetes mellitus tipo 2. 


\begin{tabular}{|c|c|c|c|}
\hline \multicolumn{4}{|c|}{ Tabla 2} \\
\hline \multicolumn{4}{|c|}{ Farmacocinética, farmacodinámica, eficacia clínica y eventos adversos de canagliflozina, dapagliflozina, empagliflozina } \\
\hline \multirow[t]{2}{*}{ Rápidez de absorción } & \multicolumn{3}{|c|}{ Administración Oral } \\
\hline & Canagliflozina & Dapagliflozina & Empagliflozina \\
\hline T max. & $1.5-2$ hrs. & 2 hrs. & $\begin{array}{l}1.33 \text { - } 3.0 \mathrm{~h} \text {, luego disminuyendo } \\
\text { en forma bifásica Moda }\end{array}$ \\
\hline Medio tiempo & $14-16$ hrs & $13 \mathrm{hrs.}$ & $\begin{array}{c}5.6-13.1 \mathrm{~h} \text { (una dosis) } \\
10.3-18.8 \mathrm{~h} \text { (múltiple dosis) }\end{array}$ \\
\hline Aclaramiento renal & $\begin{array}{c}<1 \% \text { de las dosis } \\
\text { (100 a } 200 \mathrm{mg} \text { dosis })\end{array}$ & - & $32.1-51.3 \mathrm{ML} / \mathrm{mi}$ \\
\hline $\begin{array}{l}\text { (CLR) durante } 72 \mathrm{~h} \\
\text { UGEO - } 24 \text { hrs. }\end{array}$ & $\begin{array}{l}100 \mathrm{~g} \text { ( } 100 \text { a } 300 \\
\mathrm{mg} \text { dosis })\end{array}$ & - & $71.7 \mathrm{~g}$ (50 mg de la dosis) \\
\hline $\begin{array}{c}\text { Eficacia clínica } \\
\text { Reducción de HbA1C* }\end{array}$ & $\begin{array}{c}0.74 \% \text { (Inagaki) y } 0.77 \% \\
\text { (CANTATA-M)- <P } 0.001 . \\
100 \mathrm{mg} / \mathrm{dí} \text {. }\end{array}$ & $\begin{array}{c}0.45 \% \text { (Kokui) y } 1.1196 \% \text { (Ji) } \\
\text { P<0.0001. } 10 \mathrm{mg} / \mathrm{día}\end{array}$ & $\begin{array}{c}0.66 \text { (Roden, prueba de } \\
\text { monoterapia) y } 0.83 \% \text { (Lewin) - } \\
10 \mathrm{mg} / \text { día }\end{array}$ \\
\hline Reducción de peso* & 2.5 y $2.6 \mathrm{~kg} 100 \mathrm{mg} / \mathrm{di} a$ & 2.2 y $3.2 \mathrm{~kg} 10 \mathrm{mg} / \mathrm{día}$ & 2.2 y $2.5 \mathrm{~kg} .10$ or $25 \mathrm{mg} / \mathrm{día}$ \\
\hline Lípidos & 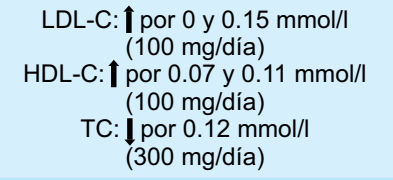 & $\begin{array}{c}\text { LDL-C: I por } 0.19 \text { y }-0.03 \mathrm{mmol} / \mathrm{l} \\
\text { HDL-C: I por } 0.16 \text { y } 0.3 \mathrm{mmol} / / \\
\text { TC: } 1 \text { por } 0.01-0.06 \mathrm{mmol} / \mathrm{I} \\
(10-\mathrm{mg} / \mathrm{dia})\end{array}$ & $\begin{array}{l}\text { LDL-C: } \text { por } 0.06 \text { y } 0.11 \mathrm{mmol} / / \\
\text { HDL-C: }: \text { por } 0.10 \text { y } 0.13 \mathrm{mmol} / \\
\text { TC: por } 0.07-0.2 \mathrm{mmol} / \mathrm{l}\end{array}$ \\
\hline $\begin{array}{l}\text { Presión de sangre } \\
\text { sistólica* } \\
\text { Eventos adversos }^{\star \star}\end{array}$ & $\begin{array}{c}\text { I por } 3.3 \text { y } 7.9 \mathrm{mmHg}(P<0.001 . \\
100 \mathrm{mg} / \mathrm{dia}) \\
\text { I por } 5.0 \mathrm{mmHg}(300 \mathrm{mg} / \mathrm{dí})\end{array}$ & I por 2.3 y 3.6 mmHg (10 mg/día) & $\begin{array}{l}\text { I por } 2.9 \text { y } 3.7 \mathrm{mmHg} \\
\text { (10 or mg/día) }\end{array}$ \\
\hline $\begin{array}{l}\text { Tracto urogenital } \\
\text { infecciones }\end{array}$ & $\begin{array}{l}\text { Poco frecuente, leve, manejado } \\
\text { con estándar tratamientos } \\
\text { y no recurrieron en ninguno } \\
\text { de los pacientes }\end{array}$ & $\begin{array}{l}\text { De gravedad leve o moderada, } \\
\text { tiende a ocurrir durante las } \\
\text { primeros o meses de la } \\
\text { terapia con dopagliflozina }\end{array}$ & $\begin{array}{l}\text { Las infecciones urogenitales } \\
\text { fueron más comunes en } \\
\text { mujeres, generalmente de leve } \\
\text { o moderada en severidad } \\
\text { y susceptible al tratamiento } \\
\text { estándar }\end{array}$ \\
\hline Cetoacidosis diabética & $\begin{array}{l}\text { Incidencias muy bajas: } 0.5 \text { por } \\
1000 \text { pacientes - años en } \\
\text { dosis diaria de } 100 \mathrm{mg} 0.8 \mathrm{en} \\
\text { dosis diaria de } 300 \mathrm{mg}\end{array}$ & - & - \\
\hline Salud ósea & $\begin{array}{l}\text { Disminución de la densidad ósea } \\
\text { y un mayor riesgo de fracturas } \\
\text { (Incidencia por } 1000 \text { pacientes - } \\
\text { años fue } 18.1 \text { para régimenes } \\
\text { de canagliflozina y } 14.2 \text { para } \\
\text { otros regímenes) }\end{array}$ & $\begin{array}{l}\text { Riesgo de fracturas } \\
\text { (Kohan et al., } 9.4 \% \text { sufrieron } \\
\text { fracturas en el grupo de } 10 \mathrm{mg} \text { ) }\end{array}$ & - \\
\hline
\end{tabular}

Tomado y Modificado de Pradhan, et SGLT2 inhibitors for diabetes and Heard failure (Downloaded free from htpp //www.jfmpc.com on Saturday, February 22.2020. IP: 189.163.121.31

\section{Referencias bibliográficas}

1. International Diabetes Federation (IDF). IDF diabetes atlas, 6thEdition; 2014. www.idf.org. Accessed 5 Sept 2016.

2.Scottish Diabetes Survey Monitoring Group. Scottish diabetes survey 2013. National Health Service (NHS) Scotland. www.diabetesinscotland.org.uk. Access Ed 5 Sept 2016.

3. Chao EC, Henry RR. SGLT2 inhibition - a novel strategy for diabetes treatment. Nat Rev Drug Discover. 2010;9:551-559.

4. Ferrannini G, Hach T, Crowe S, Sanghvi A, Hall KD . Energy balance after sodium-glucose transporter 2 inhibition. Diabetes Care. 2015;38:730-735.
5. A study to evaluate the efficacy and safety of Ertugliflozin in participants with type 2 diabetes mellitus and inadequate glycemic control on metformin monotherapy (MK-8835007). https://clinicaltrials.gov/ct2/show/NCT02033889. Access Ed 5 Sept2016.

6. Sands AT, Zambrowicz BP, Rosenstock J, LA Puerta P, Bode BW, Garg SK, et al. Sotagliflozina, a dual SGLT1 and SGLT2 inhibitor, as adjunct therapy to insulin in type 1 diabetes. Diabetes Care. 2015;38:1181-1188.

7. Ferrannini E, Ramos SJ, Salsali A, Tang W, List JF. 
Dapagliflozin monotherapy in type 2 diabetic patients with inadequate glycemic control by diet and exercise: a randomized, double-blind, placebo controlled, phase III trial. Diabetes Care. 2010;33:2217-2224.

8. Bailey CJ, Gross JL, Pieters A, Bastien A, List JF. Effect tofdapagliflozin in patients with type 2 diabetes who have inadequate glycemic control with metformin: a randomized, double-blind, placebo-controlled trial. Lancet. 2010;375:22232233.

9. Bailey CJ, Gross JL, Henricksen D, Iqbal N, Mansfield TA, List JF. Dapagliflozin as add-on to metformin in type 2 diabetes inadequately control ed with metformin: a randomized, double blind, placebo-controlled 102-week trial. BMC Med. 2013;11:43.

10.Jabbour SA, Hardy E, Sugg J, Parikh S, Study 10 Group. Dapagliflozin is effective as add-on therapy to sitagliptin with or without metformin: a 24-week, multicenter, randomized, double blind, placebo-controlled study. Diabetes Care. 2014;37:740-750.

11. Strojek K, Yoon KH, Hruba V, Elze M, Langkilde AM, Parikh S. Effect of dapagliflozin in patients with type 2 diabetes who have inadequate control with glimepiride: a randomised,24-week, double-blind, placebo-controlled trial Diabetes Obese Metab. 2011;10:928-938.

12. Nauck MA, Del Prato S, Meier JJ, Durán-García S, Rohwedder K, Elze M, Parikh SJ. Dapagliflozin versus glipizide as add-on therapy in patients with type 2 diabetes who have inadequate glycemic control with metformin: a randomized, 52-week, double-blind, active-controlled non inferiority trial. Diabetes Care. 2011;34:2015-2022.

13. Stenlof K, Cefalu WT, Kim KA, Alba M, Usiskin K, Tong C, et al. Efficacy and safety of canagliflozin monotherapy in subjects with type 2 diabetes mellitus inadequate controlled with diet and exercise. Diabetes Obese Metab. 2013;15:372382.

14. Fulcher G, Matthews DR, Perkovic V, de Zeeuw D, Mahaffey KW, Weiss R, et al. Efficacy and safety of canagliflozin used in conjunction with sulphonyl urea in patients with type 2 diabetes mellitus: a randomized control trial. Diabetes Ther.2015;6:289-302.

15. Ridderstrale M, Andersen KR, Zeller C, Kim G, Woerle HJ. Broedl UC. EMPA-REG H2H-SU trial investigators. Comparison of empagliflozin and glimepiride as add-on to metformin in patients with type 2 diabetes: a 104-week randomized, active-controlled, double-blind, Phase III trial. Lancet Diabetes Endocrinol. 2014;2:691-700.

16. Häring HU, Merker L, Seewaldt -Becker E, Weimer M, Meinicke T, Woerle HJ, et al. Empagliflozin as add-on to metformin plus sulphonyl urea in patientswithtype 2 diabetes: a 24-week, randomized, double-blind, placebo-controlled trial. Diabetes Care. 2013;36:3396-3404.

17. Rosenstock J, Jelaska A, Frappin G, Salsali A, Kim G, Woerle HJ, et al. EMPA-REG MDI Trial Investigators. Improved glucosa control with weight loss, lower insulin dose sand no increased hypoglycaemia with empagliflozin added to titrated multiple daily injections of insulin in obese inadequately controlled type 2 diabetes. Diabetes Care. 2014; 1815-1823.

18. Bolinder J, Ljunggren Ö, Kullberg J, Johansson L, Wilding J,Langkilde AM, et al. Effects of dapagliflozin on body weight ,total fat mass, and regional adipose tissue distribution in patients with type 2 diabetes mellitus with inadequate glycemic control on metformin. J Clin Endocrinol Metab. 2012;97:10201031.

19. Wilding JP, Woo V, Soler NG, Pahor A, Sugg J, Rohwedder K, et al. Dapagliflozin 006 Study Group. Long-term efficacy of dapagliflozin in patients with type 2 diabetes mellitus receiving high doses of insulin: a randomized trial. Ann Intern Med. 2012;156:405-415.

20.Henry RR, Murray AV, Marmolejo MH, Hennicken D, Ptaszynska A, List JF. Dapagliflozin, metformin XR, or both: initial pharmaco therapy for type 2 diabetes, a randomized controlled trial. Int J Clin Pract. 2012;66:446-456.

21. Sjöström CD, Sugg J, Ptaszynska A, Parikh S. Pilot analysis of the efecto of the SGLT2 inhibitor dapagliflozin on blood pressure in patients with type 2 diabetes mellitus: a pool ed analysis of placebo controlled trials. Eur Heart J. 2012;33(Suppl):S680.

22. Hach T, LambersHeerspink HJ, Pfarr E, Lund S, Ley L, Broedl UC, et al. The sodium glucose co-transporter-2 (SGLT2) inhibitor empagliflozin lowers blood pressure independent of weight or HbAlc change. Diabetología. 2012;55 Suppl1:S317. Abstract 770.

23. Cefalu WT, Leiter LA, Yoon KH, Arias P, Niskanen L, Xie J, et al. Efficacy and safety of canagliflozin versus glimepiride in patients with type 2 diabetes inadequately controlled with metformin (CANTANA-SU): 52 week results from a randomised, double-blind, Phase III non-inferiority trial. Lancet. 2013;382:941-950.

24. Zhou Y, Geng Z, Wang X, Huang Y, Shen L, Wang Y. Meta-Analysis on efficacy and safety of SGLT2 inhibitors and incretin based agents combination therapy vs. SGLT2 $\mathrm{i}$ alone or add-on to metformin in type 2 diabetes. Diabetes Metab Res Rev 2020;36(2): e3223. DOI:10.1002/dmrr. 3223.

25. Wilding JP, Charpentier G, Hollander P, González-Gálvez G, Mathieu C, Vercruysse F, et al. Efficacy and safety of canagliflozin in patients with type 2 diabetes mellitus inadequately controlled with metformin and sulphonylurea: a randomized trial. Int J Clin Pract . 2013;67(12):1267-82. doi: 10.1111/ijcp.12322.

26. Lavalle-Gonzalez FJ, Januszewicz A, Davidson J, Tong C, Qiu R, Canovatchel W, et al. Efficacy and safety of canagliflozin compared with placebo and sitagliptin in patients with type 2 diabetes on background monotherapy: a randomized trial. Diabetology. 2013;56:2582-2592.

27. Dave CV, Schneeweiss S, Kim D, Fralick M, Tong A, Patorno E. Sodium-Glucose Cotransporter-2 Inhibitors and the Risk for Severe Urinary Tract Infections: A Population Based Cohort Study. Ann Intern Med. [Epub a head of print 30 July 2019] doi:10.7326/M18-3136ea

28. Van Bommel EJ, Muskiet MH, Tonneijck L, Kramer MH, Nieuwdorp M, van Raalte DH. SGLT2 inhibition in the diabetic kidney-from mechanisms to clinical outcome. Clin J Am Soc Nephrol 2017;12(4):700-710.

29. De Nicola L, Gabbai FB, Liberti ME, Sagliocca A, Conte G, Minutolo R. Sodium/glucose cotransporter 2 inhibitors and prevention of diabetic nephropathy: targeting the renal tubule in diabetes. Am J Kidney Dis 2014;64:16-24.

30. Rosenstock J, Jelaska A, Zeller C, Kim G, Broedl UC, Woerle HJ. EMPA-REG BASAL Trial Investigators. Impact of empagliflozin added on to basal insulin in type 2 diabetes inadequately controlled on basal insulin: a 78-week, randomized, double blind, placebo-controlled trial. Diabetes 
31. Canagliflozin Cardiovascular Assessment StudyCANVAS. Available from: http://www.Acc.Org/Latest?In? Cardiology/Clinical?Trials/2017/06/12/16/25/Canvas.

Wiviott SD, Raz I, Bonaca MP, Moseazon O, Kato ET,

32. Cahn A, et al. Dapagliflozin and cardiovascular outcomes in type 2 diabetes. N Engl J Med 2019;380(4):347-357.

Pradhan, et al. SGLT-2 inhibitors for diabetes and Heart

33. failure [Downloaded free from http://www.jfmpc.com on Saturday, February 22, 2020, IP: 189.163.121.31]

34. Kosiborod M, Cavender MA, Az F, Wilding JP, Khunti $\mathbf{K}, \mathbf{H o l l} \mathbf{R W}$, et al. Lower risk of heart failure and death in patients initiated on Sodium-glucose cotransporter-2 inhibitors versus other glucose-lower ing drugs: The CVD-REAL study (Comparative effectiveness of cardiovascular outcomes in new users of sodiumglucose cotransporter-2-inhibitors). Circulation 2017;136:249-259.
35. Sha S, Polidori D, Farrell K, Ghosh A, Natarajan J, Vaccaro $\mathbf{N}$, et al. Pharmacodynamic differences between canagliflozin and dapagliflozin: results of a randomized, double-blind, crossover study. Diabetes Obes Metab. 2015;17:188-197.

36. De Nicola L, Gabbai FB, Liberti ME, Sagliocca A, Conte G, Minutolo R. Sodium/glucose cotransporter 2 inhibitors and prevention of diabetic nephropathy: targeting the renal tubule in diabetes. Am J Kidney Dis. 2014;64:16-24.

37. Kasichayanula S, Liu X, Pe Benito M. The influence of Kidney function on dapagliflozin exposure, metabolism and pharmacodynamics in healthy subjects and in patients with type 2 diabetes mellitus. Br J Clin Pharmacol. 2013;76:432-444.

38.Peters AL, Buschur EO, Buse JB, Cohan P, Diner JC, Hirsch IB. Euglycemic diabetic ketoacidosis: a potential complication of treatment with sodium-glucose cotransporter 2 inhibition. Diabetes Care. 2015;38:1687-1693.

Contribución de autoría: Dante Américo Gamarra-Gonzalez ha sido el autor del estudio, contribuyendo con concepción, búsqueda electrónica, revisión inicial, el diseño de estudio, redacción, y revisión final. Conflicto de interés: El autor no tiene conflictos de interés con la publicación de este trabajo.

Financiamiento: Autofinanciado.

Citar como: Gamarra-González DA. Papel de los inhibidores de la absorción de la glucosa SGLT2 en el tratamiento de la Diabetes Mellitus tipo2. Diagnóstico (Lima). 2020;59(2):69-76.

DOI: 10.33734/diagnostico.v59i2.220

Correspondencia: Dante Américo Gamarra González.

Correo electrónico: danteamericogg@yahoo.es danteamericogamarragonzalez@gmail.com

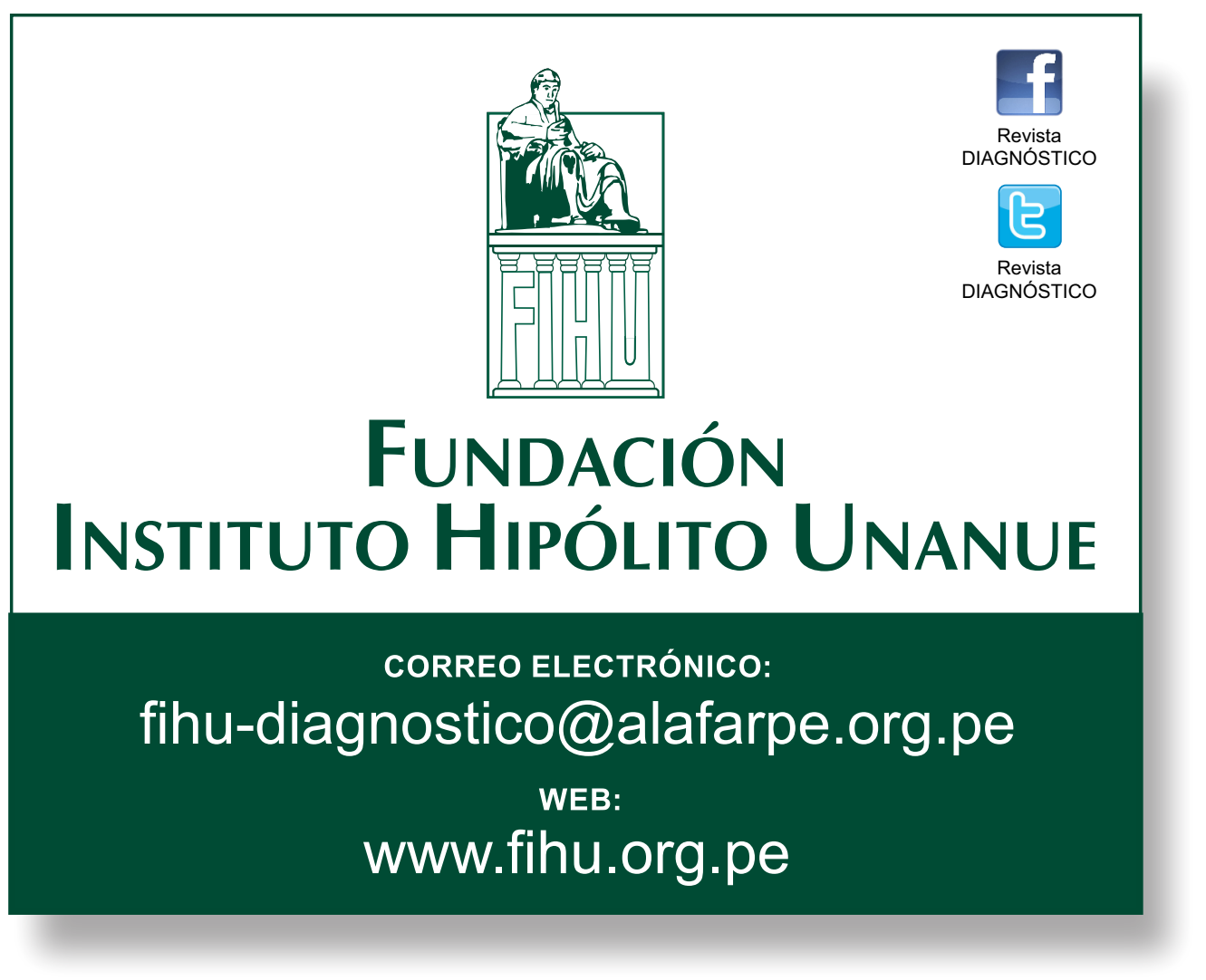

